\title{
Fossile Energiepolitik jenseits von Kyoto
}

Schleiniger, Reto ; Felder, Stefan

\begin{abstract}
In the wake of the USA's refusal to ratify the Kyoto agreement on curbing greenhouse gas emissions, this article pledges for a national energy policy beyond Kyoto, based on local external effects stemming from the combustion of fossil fuels. Due to varying external effects a national policy will differentiate energy taxes between fuels. Using Swiss estimates of external effects, it is shown that a national policy would fulfill the Kyoto goal as a secondary benefit. Moreover, a nationally based fossil fuel policy would affect the scope for an international trade of $\mathrm{CO} 2$ emission rights, as a net-buyer of emission rights will lose from participating in such a trade
\end{abstract}

DOI: https://doi.org/10.1515/1468-2516.t01-2-00003

Posted at the Zurich Open Repository and Archive, University of Zurich ZORA URL: https://doi.org/10.5167/uzh-155055

Journal Article

Published Version

Originally published at:

Schleiniger, Reto; Felder, Stefan (2003). Fossile Energiepolitik jenseits von Kyoto. Perspektiven der Wirtschaftspolitik, 4(2):197-209.

DOI: https://doi.org/10.1515/1468-2516.t01-2-00003 


\title{
Fossile Energiepolitik jenseits von Kyoto
}

\author{
Reto Schleiniger \\ Institut für Empirische Wirtschaftsforschung, Universität Zürich \\ Stefan Felder \\ Institut für Sozialmedizin und Gesundheitsökonomie, Otto-von- \\ Guericke-Universität Magdeburg
}

\section{Einführung}

Bei der Verbrennung fossiler Energieträger wird der in Öl, Gas und Kohle vorhandene Kohlenstoff an Sauerstoff gebunden. Das emittierte $\mathrm{CO}_{2}$ akkumuliert sich in der Atmosphäre und vermindert dort die Rückstrahlung des einfallenden Sonnenlichtes. Gemäß dem Intergovernmental Panel on Climate Change führt dieser Treibhauseffekt im 'business-as-usual' Szenario zu einem Anstieg der durchschnittlichen Temperaturen um 1.4 bis $5.8 \mathrm{Grad}$ Celsius bis ins Jahr $2100 .^{1}$ Dies wird wenn auch unsichere so doch erhebliche Auswirkungen auf Mensch und Natur haben.

$\mathrm{Da} \mathrm{CO}_{2}$ wie andere Treibhausgase lange in der Atmosphäre verbleibt und sich dort homogen verteilt, führt dessen Emission vollkommen unabhängig vom Emissionsstandort zu globalen Klimaveränderungen. Deshalb ist es zwingend, bei der Bewältigung des Treibhausgasproblems einen globalen Ansatz zu verfolgen, der möglichst viele Länder einbezieht. Der erste Schritt hin zu einem multilateralen Abkommen gelang 1994 als die United Nations Framework Convention on Climate Change in Kraft trat, deren explizites Ziel darin bestand, die weltweiten $\mathrm{CO}_{2}$-Emissionen $\mathrm{zu}$ stabilisieren. Drei Jahre später wurde im japanischen Kyoto ein Protokoll unterzeichnet, in welchem sich die Industriestaaten verpflichteten, die Treibhausgasemissionen im Zeitraum bis 2008-2012 um durchschnittlich fünf Prozent gegenüber $1990 \mathrm{zu}$ senken. Als sich im März 2001 die USA - sie sind mit 36 Prozent Weltanteil der größte $\mathrm{CO}_{2}$-Emittent der Vertragsparteien - unter Präsident Bush zurückzogen, geriet die Ratifizierung des Protokolls aber arg ins Stocken. Nur unter Aufweichung der Reduktionsverpflichtungen gelang es unter der Führung der 


\section{Reto Schleiniger und Stefan Felder}

EU auf zwei weiteren Konferenzen in Bonn und Marrakesch im selben Jahr, die verbliebenen Staaten für eine Ratifizierung zu gewinnen. So dürfen die Vertragsparteien neu auch die $\mathrm{CO}_{2}$-Bindung in so genannten Senken anrechnen. Für ein Land wie Russland bedeutet dies nach Schätzungen der Europäischen Kommission, dass es seine Emissionen auf der Basis von 1990 nicht einfrieren muss, sondern im Gegenteil um beinahe sechs Prozent erhöhen kann. ${ }^{2}$ Für europäische Länder wie Deutschland, Dänemark oder die Schweiz, die sich zu substanziellen Emissionsreduktionen verpflichtet haben, stellt sich die Frage, ob sie nicht die Dummen dieser Entwicklung sind. Sie produzieren ein globales Gut, von dem sie möglicherweise nicht mehr profitieren als alle anderen Länder, dessen hohe Kosten sie aber allein zu tragen haben.

Wir kommen auf der Grundlage eines allgemeinen Gleichgewichtsmodells für die Schweiz zum Ergebnis, dass sich eine Reduktion des fossilen Energieverbrauchs auch im nationalen Alleingang lohnt. Dabei berücksichtigen wir die Tatsache, dass $\mathrm{CO}_{2}$-Politik immer auch nationale Umweltpolitik ist. Denn die Verbrennung fossiler Energieträger erzeugt nicht nur den globalen Treibhauseffekt; sie betrifft ebenso die lokale Umweltqualität. Der motorisierte Verkehr produziert Lärm, verschmutzt die Luft und führt zu Todesfällen, deren Zahl von der Höhe der Energiebesteuerung beeinflusst werden kann. Unsere Ergebnisse stützen sich dabei nicht auf die viel beschworene und intensiv diskutierte doppelte Dividende (Umwelt- plus Beschäftigungsdividende), sondern ausschließlich auf die bei einer ökologischen Steuerreform zu erwartende Verbesserung der regionalen Umweltqualität. Würde Präsident Bush tatsächlich seinem Leitsatz, „Wir werden nichts tun, das unserer Wirtschaft schadet, denn meine erste Priorität sind die Menschen in Amerika", ${ }^{3}$ folgen, dann müsste er - so unsere pointierte Botschaft - das Kyoto-Protokoll ratifizieren und den Verbrauch fossiler Energie im eigenen Land einschränken.

Im nachfolgenden Abschnitt 2 bieten wir einen kleinen dogmenhistorischen Abriss über die doppelte Dividende von Umweltsteuern. Nach einem Plädoyer für die Rückbesinnung auf die erste Dividende präsentieren wir in den Abschnitten 3 und 4 am Beispiel der Schweiz die Folgen einer auf lokale Umweltgüter orientierten Energiepolitik. Abschnitt 4 beleuchtet die Friktionen eines internationalen $\mathrm{CO}_{2}$-Handels für eine nationale Umweltpolitik, während Abschnitt 5 die Ergebnisse der Arbeit zusammenfasst.

\section{Die doppelte Dividende: Eine kleine Dogmengeschichte}

Die doppelte Dividende war im letzten Jahrzehnt ohne Übertreibung das zentrale Thema des wissenschaftlichen Diskurses über die ökologische Steuerreform. Zu Beginn beschäftigten sich fast ausschließlich Umweltökonomen

2. Zitiert nach Böhringer (2002), S. 40 .

3. Zitiert nach Greiner und Michaelowa (2001), S. 236. 


\section{Fossile Energiepolitik jenseits von Kyoto}

mit dem Thema, dann aber beteiligten sich zunehmend auch Finanzwissenschaftler am Disput, ob bei einer ökologischen Steuerreform neben der Dividende für die Umwelt noch eine zweite in Form von mehr Beschäftigung oder eines verbesserten Steuersystems anfalle.

Die erste Dividende hat der britische Ökonom Pigou bereits 1912 beschrieben, zu einer Zeit also, in der Umweltverschmutzung noch kein öffentliches Thema war. Er wies darauf hin, dass bei externen Umwelteffekten eine Steuer zu einem Wohlfahrtsgewinn führe, da sie in der Lage sei, die Lücke zwischen privaten und sozialen Grenzkosten einer schädigenden Aktivität zu schließen. Die zweite Dividende ist viel jüngeren Datums. Schon sehr früh (1983) hat Binswanger vorgeschlagen, die Einnahmen einer Energieabgabe zur Finanzierung der Alterssicherung zu verwenden. Damit könne der Faktor Arbeit steuerlich entlastet und die Beschäftigung gefördert werden. ${ }^{4}$ Damit hatte Binswanger den Inhalt der doppelten Dividende exakt vorweggenommen, ohne selbst aber den Begriff zu verwenden.

Die unter Ökonomen heute gebräuchliche Terminologie der doppelten Dividende lieferte erst Goulder im Jahre 1995. In ihrer starken Form bedeutet die doppelte Dividende, dass "the revenue neutral substitution of the environmental tax for typical or representative distortionary taxes involves a zero or negative gross cost". ${ }^{\prime 5}$ Die Substanz dieser Definition liegt darin, dass die ökologische Steuerreform auch dann keine Kosten verursacht oder sogar Erträge abwirft, wenn man von ihrer ökologischen Wirkung absieht. Zwar halten sich in der Literatur noch andere Definitionen wie bspw. die Reduktion der Arbeitslosigkeit, jene von Goulder ist aber, da sie sich auf die Gesamtwohlfahrt bezieht, die allgemeinste Formulierung der doppelten Dividende.

Warum nun aber soll die ökologische Steuerreform, die umweltbelastende Aktivitäten besteuert und die Einnahmen zur Reduktion der Lohnsteuer verwendet, über den Umweltnutzen hinaus Vorteile für die Gesamtwohlfahrt bringen? Man ist zunächst versucht, die Ramsey-Regel zu bemühen. In den meisten Industriestaaten ist die Besteuerung von Arbeit bekanntlich höher als jene von Energie. Da die Verzerrung einer Steuer mit dem Steuersatz quadratisch zunimmt, sollte es möglich sein, die Ineffizienz des Steuersystems insgesamt zu reduzieren, indem man den niedrigen Steuersatz auf Energie anhebt und mit den zusätzlichen Einnahmen die hohe Besteuerung der Arbeit senkt. Die Erhöhung der Energiesteuer wird zwar die Verzerrung im Energiemarkt erhöhen, die Reduktion der stark verzerrenden Steuer auf das Arbeitseinkommen kann diesen Effekt jedoch mehr als kompensieren. Dies trifft jedenfalls dann zu, wenn die Preiselastizität auf dem Energiemarkt jene auf dem Arbeitsmarkt nicht übersteigt. Pearce hat noch 1991 genau diesen Aspekt betont, als er begründete, weshalb eine einseitige $\mathrm{CO}_{2}$-Steuer zur Bekämpfung des globalen Treibhauseffektes für ein einzelnes Land vorteilhaft

4. Vgl. Abschnitt 5.4.4 in Binswanger etal. (1983).

5. Goulder (1995), S. 4. 


\section{Reto Schleiniger und Stefan Felder}

sei. ${ }^{6}$ Seine Bezugnahme auf die doppelte Dividende war insofern konsequent, als der Nutzen aus der Steuer global ist, die Kosten jedoch im Inland anfallen. Ein Alleingang kann sich daher für ein einzelnes kleines Land nur dann lohnen, wenn die Kosten im Sinne von Goulder negativ sind.

Ökologischen Interessengruppen war die These der doppelten Dividende sehr willkommen. Ein erhebliches Legitimationsproblem eines nationalen Alleinganges in der $\mathrm{CO}_{2}$-Politik schien gelöst und ad hoc Rechtfertigungen wie globale Solidarität oder der so genannte ,first mover advantage ${ }^{t}-$ die vorauseilende Nation verschafft sich einen technologischen Vorsprung - entbehrlich. Grüne Politiker konnten sich als Finanzpolitiker profilieren. Wirtschaftsvertreter dagegen gerieten in argumentative Verlegenheit und mussten sich den Vorwurf gefallen lassen, mit ihrem Widerstand gegen eine ökologische Steuerreform eine effiziente Gestaltung des Steuersystems zu verhindern. Schließlich nahm auch die Politikberatung die Idee der doppelten Dividende auf und setzte auf die Vorteile einer ökologischen Steuerreform für den Arbeitsmarkt. ${ }^{7}$

So verlockend die Vorstellung einer doppelten Dividende war, so wenig konnte sie einer wissenschaftlich fundierten Analyse standhalten. Bovenberg und de Mooij (1994) versetzten ihr in einem viel beachteten Artikel nicht gerade den Todesstoß, fügten ihr aber doch schweren Schaden zu. Sie machten darauf aufmerksam, dass das partialanalytische Modell, auf dem die Behauptung gründet, die Interdependenz zwischen Energie- und Arbeitsmarkt nicht berücksichtige und deshalb zu voreiligen Schlüssen führe. In der Tat kann die Auswirkung einer Steuerreform auf die gesamtwirtschaftliche Effizienz nur im Rahmen eines allgemeinen Gleichgewichtsmodells abschließend beurteilt werden, das insbesondere die Steuerüberwälzungen vom Energie- in den Arbeitsmarkt einbezieht. In einem einfachen theoretischen Modell zeigten sie, dass die ökologische Steuerreform ohne Berücksichtigung der Umweltwirkungen in der Regel zu einem Wohlfahrtsverlust führt. Typischerweise steigt mit Erhöhung der Energiesteuer das allgemeine Preisniveau. Trotz Rückverteilung der Steuereinnahmen kommt es damit zu einer Reduktion der realen Löhne nach Steuern. So nützt es den Arbeitnehmern wenig, wenn die Löhne durch eine Reduktion der Lohnnebenkosten z. B. um zwei Prozent zunehmen, gleichzeitig aber das allgemeine Preisniveau wegen der Energiesteuer um drei Prozent steigt.

Abbildung 1 verdeutlicht das Argument. Eine Erhöhung der Energiesteuer von $t_{0}$ auf $t_{1}$ verringert den Reallohn, da die Kaufkraft um $E_{0} \cdot\left(t_{1}-t_{0}\right)$ zurückgeht. Die Rückverteilung der zusätzlichen Steuereinnahmen an die Arbeit entspricht der Differenz der Flächen C und B [Energiesteueraufkommen nach Steuererhöhung $(=\mathrm{A}+\mathrm{C})$ minus Steueraufkommen vor Steuererhöhung $(=\mathrm{A}+\mathrm{B})]$. Der Betrag C-B reicht aber nicht aus, um den

6. Pearce (1991, S. 940). Kirchgässner (1998) bezeichnete Pearces Arbeit als letzte, welche die Existenz einer doppelten Dividende 'unhinterfragt unterstellt'.

7. So zum Beispiel Meier et al. (1998), S. 24, 40 und 52. 


\section{Fossile Energiepolitik jenseits von Kyoto}

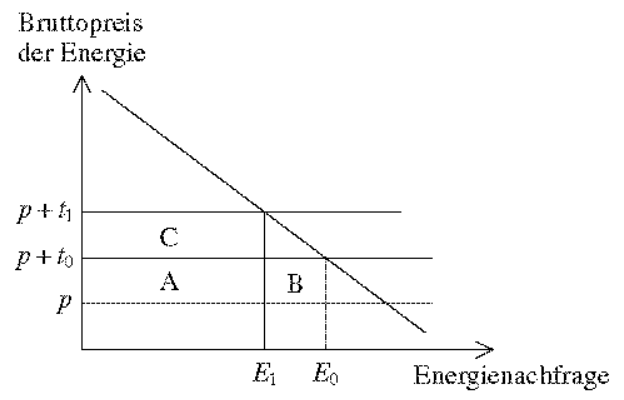

Abbildung 1 Die Wirkung einer Steuer auf Energie- und Arbeitsmarkt

Kaufkraftverlust zu kompensieren, so dass der reale Nettolohn nach Steuern sinkt. Keine Wirkung auf den Arbeitsmarkt wäre nur dann zu erwarten, falls die rückverteilte Summe dem Kaufkraftverlust entspräche. Dies wäre nur bei einer Ausgangssituation ohne Energiesteuer und einer marginalen Steuererhöhung oder aber bei vollkommen unelastischer Energienachfrage der Fall. Während Bovenberg und de Mooij das Verdienst zukommt, auf das Problem der Steuerüberwälzung hingewiesen zu haben, schien anderen Finanzwissenschaftlern die ganze Diskussion geradezu trivial. So machten Gottried und Wiegard (1995) etwas indigniert darauf aufmerksam, dass eine ökologische Steuerreform nur dann das Ausmaß der Steuerineffizienz reduzieren und damit zu einer doppelten Dividende führen könne, wenn das Steuersystem im Ausgangspunkt nicht bereits optimiert sei. Gerade im Modell von Bovenberg und de Mooij ist dies aber der Fall. Offensichtlich kann jedoch ein bereits optimiertes Steuersystem über eine Reform nicht weiter verbessert werden, sondern führt im Gegenteil zwangsläufig zu einem Wohlfahrtsverlust.

In der Folge erschienen eine Reihe von Beiträgen, welche die Möglichkeit einer doppelten Dividende für verschiedene Fälle aufzeigten, in denen das Ausgangssteuersystem nicht zweitbest ist. ${ }^{8}$ Stellvertretend sei eine Arbeit von Koskela et al. (2001) genannt, bei der ein zu hoher, gewerkschaftlich fixierter Reallohn im Ausgangsgleichgewicht Arbeitslosigkeit erzeugt. Da die ökologische Steuerreform den Reallohn senkt, reduziert sie gleichzeitig die Ineffizienz des bestehenden Steuersystems. Die ökologische Steuerreform dient sozusagen als wirtschaftspolitisches Ersatzinstrument für eine Reallohnsenkung. Es stellt sich allerdings die Frage, weshalb die Therapie nicht direkt beim Problem eines zu hohen Reallohns ansetzen kann.

Im Lichte der Forschungsergebnisse wird es zunehmend schwierig, eine nationale $\mathrm{CO}_{2}$-Politik des Alleingangs zu rechtfertigen. Das Argument der doppelten Dividende hat, abgesehen von einzelnen Nebenschauplätzen, ausgedient. Sind damit aber die Staaten, die das Kyoto-Protokoll ratifiziert haben, tatsächlich die Dummen? Die Antwort ist nein. Der Grund hierfür ist 


\section{Reto Schleiniger und Stefan Felder}

so einfach wie zwingend: Beim Verbrauch fossiler Energie wird nicht nur $\mathrm{CO}_{2}$ emittiert, sondern es werden eine Reihe von anderen externen Effekten verursacht, die aber im Unterschied zum Treibhauseffekt regionaler Natur sind. So stammen praktisch alle Emissionen von Stickstoffoxiden, Schwefeldioxid, Kohlenmonoxid und Staub, insbesondere der karzinogene Dieselruß, sowie ein großer Teil der Emissionen aus flüchtigen organischen Verbindungen aus der Verbrennung fossiler Energieträger. ${ }^{9}$ Eine Reduktion des fossilen Energieverbrauchs würde die lokalen Belastungen deutlich vermindern und zu einer Wohlfahrtsverbesserung führen, da dieser Nutzen im Unterschied zur $\mathrm{CO}_{2}$-Minderung zum größten Teil innerhalb der Landesgrenzen anfällt.

Die Begründung für eine nationale fossile Energiepolitik bedarf demnach nicht länger der doppelten Dividende. Es reicht aus, sich auf die erste Dividende zu beziehen. Mit dieser Argumentationslinie geht eine Akzentverschiebung einher, weg vom globalen Treibhauseffekt hin zu lokalen externen Effekten des fossilen Energieverbrauchs.

\section{Fossile Energiebesteuerung: Beispiel Schweiz}

Die Schweizer Regierung gab in den neunziger Jahren eine Reihe von Studien in Auftrag, um Aufschluss über die national anfallenden externen Kosten beim Verbrauch fossiler Energie zu erhalten. ${ }^{10}$ Tabelle 1 fasst die Ergebnisse

Tabelle 1 Preise und Kostenzuschläge für fossile Energieträger in der Schweiz

\begin{tabular}{|c|c|c|c|c|c|}
\hline & & $\begin{array}{l}\text { Benzin } \\
0.01 \mathrm{CHF} / 1\end{array}$ & $\begin{array}{l}\text { Diesel } \\
0.01 \mathrm{CHF} / 1\end{array}$ & $\begin{array}{l}\text { Heizöl } \\
\text { CHF/100l }\end{array}$ & $\begin{array}{l}\text { Erdgas } \\
0.01 \mathrm{CHF} / \mathrm{kWh}\end{array}$ \\
\hline $\begin{array}{l}\text { gegenwärtiger } \\
\text { Preis }\end{array}$ & $\begin{array}{l}\text { Nettopreis } \\
\text { spezifische Steuern } \\
\text { Mehrwertsteuer } \\
\text { Bruttoverkaufspreis }\end{array}$ & $\begin{array}{l}55 \\
74 \\
11 \\
140\end{array}$ & $\begin{array}{l}53 \\
77 \\
11 \\
140\end{array}$ & $\begin{array}{l}42 \\
1 \\
3 \\
46\end{array}$ & $\begin{array}{l}3.88 \\
0.02 \\
0.29 \\
4.19\end{array}$ \\
\hline Zuschläge & $\begin{array}{l}\text { Luftverschmutzung } \\
\text { Verkehrsunfälle } \\
\text { Verkehrslärm } \\
\text { Straßenbau und } \\
\text {-unterhalt } \\
\text { Total Zuschläge }\end{array}$ & $\begin{array}{l}40-109 \\
68-99 \\
14 \\
37 \\
159-260\end{array}$ & $\begin{array}{l}79-202 \\
20-31 \\
18 \\
26 \\
144-278\end{array}$ & $\begin{array}{l}36-91 \\
- \\
- \\
- \\
36-91\end{array}$ & $\begin{array}{l}2.43-6.20 \\
- \\
- \\
- \\
2.43-6.20\end{array}$ \\
\hline Pigou-Preis ${ }^{\text {a) }}$ & & $214-315$ & $196-330$ & $77-132$ & $6.31-10.08$ \\
\hline
\end{tabular}

${ }^{a}$ Da die Zuschläge auf der Basis des Nettopreises berechnet wurden, ergibt sich der Pigou-Preis jeweils als Summe von Nettopreis und Zuschlag.

9. Ekins (1996), S. 13.

10. Eine Zusammenfassung dieser Studien findet sich in Infras et al. (1996). 


\section{Fossile Energiepolitik jenseits von Kyoto}

als Kostenzuschläge für die vier Energieträger Benzin, Diesel, Heizöl und Erdgas zusammen. Kohle ist als Energieträger in der Schweiz praktisch vernachlässigbar und wird deshalb nicht aufgeführt. Da Kostenschätzungen zwangsläufig mit einer gewissen Unschärfe behaftet sind, geben wir für jeden Energieträger jeweils eine untere und eine obere Grenze an. ${ }^{11}$

Bei allen Energieträgern entstehen Kosten im Zusammenhang mit der Luftverschmutzung. Dabei sind neben den Gebäude- und Waldschäden vor allem die Gesundheitskosten und darunter wiederum die Kosten von frühzeitigen Todesfällen von Bedeutung. Die Abschätzung der Todesfälle stützt sich auf epidemiologische Studien aus den USA, die einen signifikanten Zusammenhang zwischen der Luftbelastung (gemessen mit dem Leitschadstoff PM10) und frühzeitigen Todesfällen ausweisen. ${ }^{12}$ Die auf dieser Grundlage für die Schweiz geschätzte Zahl jährlicher frühzeitiger, auf verkehrsbedingte Luftverschmutzung zurückzuführender Todesfälle beträgt 2100. Die Lebenserwartung reduziert sich durchschnittlich um 12.7 Jahre, die wir insgesamt mit einem Verlust von 2-3 Mio. Franken bewerten. ${ }^{13}$

Neben der Luftbelastung entstehen beim Verbrauch von Treibstoffen Kosten in Zusammenhang mit Unfällen - auch hier machen die Todesfälle den größten Kostenanteil aus ${ }^{14}$-, Lärm sowie Bau und Unterhalt der Straßen.

Die in der Tabelle 1 aufgeführten Pigou-Preise liegen trotz der bestehenden, teilweise hohen fiskalischen Belastung deutlich über den derzeitigen Bruttoverkaufspreisen. So kostet der Liter Benzin rund 1.40 Franken, ein kostendeckender Preis müsste sich aber zwischen 2.14 und 3.15 bewegen. Ähnliches gilt für Diesel. Auch das effiziente Preisband von Heizöl und Erdgas liegt um mehr als das Doppelte über den aktuellen Marktpreisen. Fossile Energieträger sind also aus volkswirtschaftlicher Sicht zu billig. Das heißt, auch ohne Beachtung des Treibhauseffektes ist der Verbrauch fossiler Energie zu hoch und seine Einschränkung könnte über die Verbesserung der lokalen Umweltqualität die nationale Wohlfahrt erhöhen.

Die Existenz substanzieller externer Effekte auf nationaler Ebene wird zuweilen mit dem Argument in Zweifel gezogen, die bestehenden nationalen politischen Institutionen würden selbst für eine Internalisierung der externen Effekte sorgen. Ohne die politische Ökonomie der Umweltregulierung im Detail zu bemühen, ${ }^{15}$ scheint uns diese Argumentation etwas naiv. Wir leben trotz bestehender Institutionen nicht in der besten aller Welten. Gutorganisierte Interessengruppen verhindern, dass ineffiziente Importzölle auf landwirtschaftliche Produkte und Subventionen im Kohlebergbau

11. Eine umfassende Darstellung aller Berechnungen dieses Abschnitts findet sich in Felder und Schleiniger (2002a).

12. Vgl. Ecoplan (1996), S. K-6.

13. Ecoplan (1996), S. K-9.

14. Die eigenen Kosten eines Verkehrsunfalls sowie weitere durch Versicherung gedeckte Kosten wurden bei der Bestimmung der externen Kosten ausgeschlossen.

15. Vgl. dazu aber im Detail Schleiniger und Felder (1998) und Felder und Schleiniger (2002b). 


\section{Reto Schleiniger und Stefan Felder}

abgeschafft werden. Aus demselben Grund können wir auch nicht zwingend damit rechnen, dass externe Effekte im Umweltbereich internalisiert werden.

Es gibt nun zwei Möglichkeiten, die lokalen externen Effekte bei einer fossilen Energiepolitik zu berücksichtigen. Man könnte wie Ekins (1996) den Nutzen einer lokalen Umweltverbesserung als, secondary benefit ${ }^{t}$ einer $\mathrm{CO}_{2}$-Reduktion einbeziehen. Aus nationaler Sicht ist es allerdings angemessener, den umgekehrten Weg zu beschreiten, nämlich die lokalen externen Effekte $\mathrm{zu}$ internalisieren und die Wirkungen auf die $\mathrm{CO}_{2}$-Emissionen als ,secondary benefit' zu verstehen.

Eine Untersuchung mit Hilfe eines berechenbaren Gleichgewichtsmodells (vgl. Felder und Schleiniger, 2002a) kommt zum Ergebnis, dass eine ökologische Steuerreform bestehend aus einer Internalisierung der lokalen externen Effekte und der Verwendung der dadurch erzielten Einnahmen zur Reduktion der Arbeitsbesteuerung die Wohlfahrt der Schweizer um 1 bis 5 Milliarden Franken erhöht. Dies entspricht 0.25 bis 1.25 Prozent des Bruttoinlandproduktes. ${ }^{16}$ Der mit der Internalisierung verbundene Rückgang des Verbrauchs fossiler Energie führt gleichzeitig zu einer Reduktion der $\mathrm{CO}_{2}$-Emissionen um 30 bis 50 Prozent.

Diese Ergebnisse sind zwar stark von den verwendeten Parametern abhängig, Sensitivitätsanalysen zeigen aber, dass die internationalen $\mathrm{CO}_{2}$ Verpflichtungen der Schweiz ${ }^{17}$ durch eine nationale fossile Energiepolitik problemlos eingehalten werden. Mit anderen Worten bewirkt die Internalisierung der eigenen externen Effekte des fossilen Energieverbrauchs für die Schweiz die Erfüllung der Kyoto-Verpflichtung ganz nebenbei und kostenlos. Aufgrund der vergleichbaren Umweltprobleme in Europa ist davon auszugehen, dass auch andere europäische Staaten durch eine konsequente Internalisierungsstrategie den eingegangenen $\mathrm{CO}_{2}$-Verpflichtungen nachkommen könnten.

Es stellt sich nun die Frage, ob eine solche nationale Internalisierungsstrategie in der Wahl der Instrumente überhaupt von einer $\mathrm{CO}_{2}$-Abgabepolitik zu unterscheiden ist, da beide Ansätze zu einer Erhöhung der Energiepreise führen. Wiederum zeigt sich aber für die Schweiz, dass die Steuersätze zur Internalisierung der nationalen Externalitäten stark von den Steuersätzen einer $\mathrm{CO}_{2}$-Politik abweichen. Bei einer uniformen $\mathrm{CO}_{2}$-Steuer werden die Treibstoffe im Vergleich zu den Brennstoffen viel weniger belastet als bei der Internalisierungsstrategie. Dies erklärt sich dadurch, dass die Treibstoffe weit höhere externe Effekte verursachen als die Brennstoffe, gleichzeitig aber

16. Bei einer pauschalen Rückverteilung reduziert sich dieser Gewinn um 25 bis 66 Prozent. Dies ist ein Hinweis darauf, wie wichtig es ist, dass die zusätzlichen Steuereinnahmen zur Reduktion bestehender Steuern eingesetzt werden.

17. Im Rahmen des Kyoto-Protokolls hat sich die Schweiz wie auch die EU - ohne Berücksichtigung von Senken - zu einer achtprozentigen Reduktion verpflichtet. Im $\mathrm{CO}_{z^{-}}$ Gesetz hat man dieses Reduktionsziel auf zehn Prozent erhöht. 


\section{Fossile Energiepolitik jenseits von Kyoto}

die gegenwärtige Besteuerung diesen unterschiedlichen externen Effekten zu wenig Rechnung trägt.

Exemplarisch deutlich wird dies beim Vergleich von Diesel und Heizöl, deren $\mathrm{CO}_{2}$-Intensität aufgrund der gleichen chemischen Zusammensetzung identisch ist. Eine $\mathrm{CO}_{2}$-Steuer belastet Diesel und Heizöl gleich stark. Bei einer Internalisierungsstrategie steigt dagegen der Dieselpreis beinahe doppelt so stark wie der Heizölpreis.

Eine an den externen Effekten ausgerichtete fossile Energiepolitik sollte also die verschiedenen fossilen Energieträger auch unterschiedlich stark besteuern. Auf der Ebene der vier Energieträger ist dies insofern kein Problem, als dass bereits heute eine fiskalische Differenzierung besteht. Eine eigentliche $\mathrm{CO}_{2}$-Politik drängte sich erst dann auf, wenn nach erfolgter Internalisierung die festgelegten Emissionsreduktionen nicht erreicht würden. Von einem solchen zweiten Schritt sind wir aber in Europa angesichts der bedeutenden lokalen Externalitäten und gleichzeitig bescheidenen $\mathrm{CO}_{2}$-Reduktionszielen noch weit entfernt.

\section{Internationaler $\mathrm{CO}_{2}$-Handel}

Die Unterscheidung zwischen globalen und regionalen externen Effekten beim Verbrauch fossiler Energie hat weitreichende Konsequenzen für die Beurteilung der so genannten flexiblen Mechanismen, welche ins KyotoProtokoll aufgenommen wurden. Diese Regelungen sehen ihrem Grundsatz nach einen internationalen Handel von national zugeteilten Emissionsrechten vor.

Gerade Ökonomen setzen sich für eine derartige Flexibilisierung der Emissionsziele auf Länderebene ein, weil sie die Emissionen dort reduzieren würde, wo die Vermeidungskosten am geringsten wären. Bei einem weltweit einheitlichen Emissionspreis, so das Argument, würden auch die Grenzvermeidungskosten über Länder hinweg ausgeglichen und damit die gesamten Vermeidungskosten minimiert.

Die effizienzsteigernde Wirkung von Handel ist unumstritten und bedarf keiner weiteren Begründung. Die Argumente, die gegen eine solche Flexibilisierung der Kyoto Verpflichtungen angeführt werden, sind schwach. So überzeugt bspw. die Folgerung, die reichen Staaten könnten sich von ihrer Verpflichtung durch einen unmoralischen Ablasshandel loskaufen, wenig. Dabei wird übersehen, dass Handel kein Nullsummenspiel ist, sondern alle Handelspartner besser stellen kann. Wenn man in diesem Zusammenhang Gerechtigkeitsüberlegungen einbringen will, so sollten sich diese ganz im Sinne von Coase auf die Verteilung der Verschmutzungsrechte, nicht aber auf deren Tausch beziehen.

Unser Argument gegen den Handel von Emissionsrechten basiert hingegen auf Effizienzüberlegungen. Wenn es substanzielle lokale externe Effekte des Verbrauchs fossiler Energie gibt, dann bewegen wir uns zwangsläufig in der 


\section{Reto Schleiniger und Stefan Felder}

Welt des Zweitbesten. Handelsvorteile, die sich in der erstbesten Welt unzweideutig ergeben, sind in der zweitbesten Welt aber unsicher. Der internationale Handel von $\mathrm{CO}_{2}$-Rechten würde $\mathrm{zu}$ falschen Preisen stattfinden und könnte daher nicht zu einer optimalen internationalen Allokation des global fixierten - fossilen Energieverbrauchs führen. Ein Land, welches Emissionsrechte kauft, erhöhte nicht nur seinen fossilen Energieverbrauch, sondern auch die externen Kosten. Umgekehrt würden in einem Verkäuferland mit dem verminderten Energieverbrauch die externen Effekte eingeschränkt.

Die Verzerrung der internationalen Energieallokation durch den Handel mit Emissionsrechten wird um so größer sein, je unterschiedlicher die Bewertung der lokalen Externalitäten unter den Handelspartnern ist. ${ }^{18}$ Insbesondere aufgrund der hohen Einkommenselastizität des Gutes Umwelt ist die Bewertung von Umweltbelastungen in reichen Ländern viel höher als in ärmeren Regionen. ${ }^{19}$

Eine numerische Abschätzung des globalen Effizienzverlustes von internationalem $\mathrm{CO}_{2}$-Handel ist äußerst schwierig. Dazu müssten die externen Kosten in allen Handelsländern bekannt sein. Für die Schweiz lassen sich aber die Wohlfahrtswirkungen der Einführung eines internationalen Handels von $\mathrm{CO}_{2}$-Eigentumsrechten mit Hilfe der Schätzungen über die externen Kosten ermitteln. So zeigt sich am Beispiel Benzin in Tabelle 1, dass die Konsumenten für eine zusätzliche Einheit nur 1.40 Franken zahlen, diese Einheit aber gesamtwirtschaftliche Kosten von 2.14 bis 3.15 Franken verursacht. Durch die Einführung eines internationalen Zertifikatshandels würde daher die Gefahr entstehen, dass die Schweiz fossile Energie kauft, die mehr kostet, als dass sie wert ist.

Abbildung 2 verdeutlicht graphisch, wie die Schweiz beim Zukauf von Zertifikaten Gefahr liefe, Wohlfahrt einzubüßen. Ausgangspunkt ist eine Situation ohne internationalen Handel mit eingeschränktem Energieverbrauch $\bar{E}$. Nach Eröffnung des $\mathrm{CO}_{2}$-Handels kauft die Schweiz Verschmutzungsrechte hinzu und weitet den Energieverbrauch bis $E$ aus, da die private Nachfrage bei $\bar{E}$ oberhalb des internationalen Energiepreises $P_{E}$ verläuft, der den Zertifikatspreis einschließt. Wegen der bedeutenden nationalen Externalitäten verläuft die soziale Wertschätzung aber weit unterhalb der privaten Nachfrage nach Energie. Der Anstieg des Energieverbrauchs führt daher zu einem Wohlfahrtsverlust in der Höhe der markierten Fläche. Die Abbildung zeigt auch, dass dieser Verlust vom Preis der fossilen Energie abhängig ist, da dieser das Ausmaß der Ausweitung des Energieverbrauchs bestimmt.

18. Ausführlich dazu und zu den numerischen Resultaten in diesem Abschnitt vgl. Schleiniger (2001).

19. Die mit dem Volkseinkommen zunehmende Wertschätzung der Umwelt hat allerdings auch dazu geführt, dass in den reicheren Ländern Umweltgesetze in Kraft traten, die die lokale Verschmutzung eindämmten. Dennoch gibt es auch in diesen Ländern noch heute bedeutende lokale Umweltbelastungen, die - wie die Schätzungen aus dem letzten Abschnitt zeigen - hohe externe Kosten verursachen. 


\section{Fossile Energiepolitik jenseits von Kyoto}

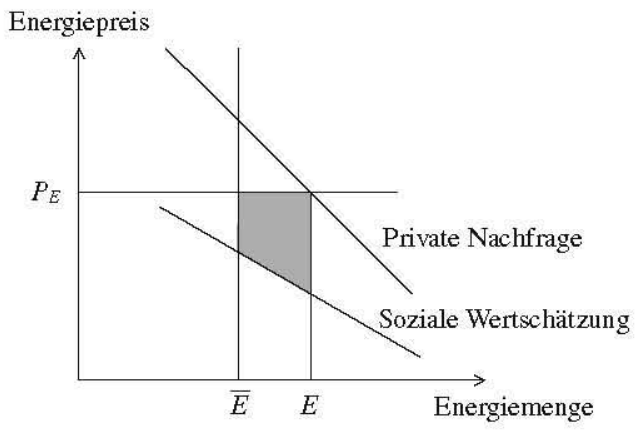

Abbildung 2 Der Wohlfahrtsverlust beim Kauf von Emissionsrechten

Globalen Gleichgewichtsmodellen auf der Grundlage der Kyoto-Verpflichtungen zufolge würden die Preise von Emissionsrechten tatsächlich so niedrig ausfallen, ${ }^{20}$ dass die Schweiz zu den Käuferländern zählte. Nach unseren Berechnungen ergibt sich bei einem Preis für Emissionsrechte von 50 Franken pro Tonne Kohlenstoff daher ein Wohlfahrtsverlust zwischen 0.4 und 1.2 Milliarden Franken, abhängig davon, ob das Minimum oder das Maximum der Schätzung der lokalen Externalitäten berücksichtigt wird. Dies entspricht zwischen einem und drei Promille des BIP. Einem Land wie der Schweiz ist daher abzuraten, bei den gegebenen nationalen externen Effekten des fossilen Energieverbrauchs an einem internationalen $\mathrm{CO}_{2}$-Handel teilzunehmen. Die Schweiz würde dadurch nicht nur zusätzliche fossile Energie, sondern gleichzeitig auch zusätzliche lokale Umweltverschmutzung einkaufen.

\section{Fazit}

Wie man auch zum Rückzug der USA vom Kyoto-Abkommen stehen mag: Im Entscheid der USA liegt immerhin die Chance, dass die anderen Länder in der fossilen Energiepolitik ihren Blick von der internationalen zurück auf die nationale Ebene richten. Eine nationale Energiepolitik jenseits von Kyoto, die bei den lokalen externen Effekten ansetzt, verspricht jedenfalls einen nationalen Wohlfahrtsgewinn. Eine solche fossile Energiepolitik des Alleingangs würde die Steuern nach Energieträger differenzieren, da sich die externen Effekte je nach Brenn- und Treibstoff unterscheiden.

Eine Ausrichtung auf die lokalen externen Effekte hätte auch Konsequenzen für den internationalen $\mathrm{CO}_{2}$-Handel, wie er im Kyoto-Protokoll skizziert ist. Solange die Bruttopreise der Energieträger ihre externen Kosten nicht decken, sollte ein Land, um Wohlfahrtsverluste zu vermeiden, keine

20. Weyant (1999) und v.a. Böhringer (2002), der die Auswirkungen des Einbezugs von Senken auf den Zertifikatspreis berücksichtigt. 


\section{Reto Schleiniger und Stefan Felder}

$\mathrm{CO}_{2}$-Emissionsrechte zukaufen. Bei den zu erwartenden Zertifikatspreisen ist davon auszugehen, dass die meisten Industrieländer tatsächlich Nettokäufer sein werden. In diesem Falle würde es sich für diese Länder nicht lohnen, am $\mathrm{CO}_{2}$-Handel zu partizipieren.

Zwar betreffen die hier vorgestellten numerischen Ergebnisse einer nationalen fossilen Energiepolitik nur die Schweiz. Sie dürften aber auch auf andere entwickelte Länder zutreffen, die mit ähnlichen lokalen Umweltproblemen konfrontiert sind. Ein Indiz dafür, wie wichtig die lokale Umweltqualität ist, geben gerade die Verhältnisse in den USA. Auch wenn die Vereinigten Staaten sich nicht am Kyoto-Protokoll beteiligen, haben mehr als die Hälfte ihrer Bundesländer eine eigene $\mathrm{CO}_{2}$-Politik bzw. fossile Energiepolitik formuliert.

\section{Literaturverzeichnis}

Binswanger, H. C. et al. (1983), Arbeit ohne Umweltzerstörung, S. Fischer, Frankfurt am Main.

Böhringer, C. (2002), Climate Politics From Kyoto to Bonn: From Little to Nothing?, The Energy foumal 23(2), 51-71.

Bovenberg, A. L. (1999), Green Tax Reform and the Double Dividend: an Updated Reader's Guide, International Tax and Public Finance 6(3), 421-443.

Bovenberg, A. L. and R. A. de Mooij (1994), Environmental Levies and Distortionary Taxation, American Economic Review 94(4), 1085-1089.

Ecoplan (1996), Monetarisierung der verkehrsbedingten externen Gesundheitskosten, Dienst für Gesamtverkehrsfragen, Bundesamt für Energie Nr. 272, Bern.

Ekins, P. (1996), The secondary Benefits of CO2 Abatement: How Much Emission Reduction do they Justify?, Ecological Economics 16, 13-24.

Felder, S. and R. Schleiniger (2002a), National $\mathrm{CO}_{2}$ Policy and Externalities: Some General Equilibrium Results for Switzerland, Energy Economics 24(5), 509-522.

Felder, S. and R. Schleiniger (2002b), Environmental Tax Reform: Efficiency and Political Feasibility, Ecological Economics 42, 107-116.

Goulder, L. H. (1995), Environmental Taxation and the 'Double Dividend': A Reader's Guide, International Tax and Public Finance 2, 157-183.

Gottfried, P. und W. Wiegard (1995), Wunderwaffe Ökosteuern? Eine finanzwissenschaftliche Betrachtung, Wist 10, 500-508.

Greiner, S. und A. Michaelowa (2001), Bushs Absage an das Kioto-Protokoll - wird die EU Lokomotive der globalen Klimapolitik?, Wirtschaftsdienst IV, 236-239.

Infras, Econcept und Prognos (1996), Die vergessenen Milliarden, Paul Haupt, Bern, Stuttgart und Wien.

Intergovernmental Panel on Climate Change (2001), Climate Change 2001: Impacts, Adaptation, and Vulnerability, Summary for Policymakers.

Kirchgässner, G. (1998), Ökologische Steuerreform: Utopie oder realistische Alternative?, Zeitschrift für Wirtschafts- und Sozialwissenschaften 256(6), 279-319.

Koskela, E., R. Schöb und H.-W. Sinn (2001), Green Tax Reform and Competitiveness, German Economic Review 2(1), 19-30. 


\section{Fossile Energiepolitik jenseits von Kyoto}

Meier, R., Messerli, P. und G. Stephan (Hrsg.) (1998), Ökologische Steuerreform für die Schweiz, Rüegger, Chur/Zürich.

Pearce, D. (1991) The Role of Carbon Taxes in Adjusting to Global Warming, The Economic fournal 101, 938-948.

Schleiniger, R. und S. Felder (1998), Effizienz und politische Durchsetzbarkeit: Die Verwendung der Einnahmen einer CO2-Abgabe in der Schweiz, Schweizerische Zeitschrift für Volkswirtschaft und Statistik 134(3), 355-367.

Schleiniger, R. (2001), Global $\mathrm{CO}_{2}$-Trade and Local Externalities, University of Zurich, Institute for Empirical Research in Economics, Working Paper No. 77.

Weyant, J. P. (ed.) (1999), The Costs of the Kyoto Protocol: A Multi-Model Evaluation, The Energy Journal, Special Issue.

Abstract: In the wake of the USA's refusal to ratify the Kyoto agreement on curbing greenhouse gas emissions, this article pledges for a national energy policy beyond Kyoto, based on local external effects stemming from the combustion of fossil fuels. Due to varying external effects a national policy will differentiate energy taxes between fuels. Using Swiss estimates of external effects, it is shown that a national policy would fulfil the Kyoto goal as a secondary benefit. Moreover, a nationally based fossil fuel policy would affect the scope for an international trade of $\mathrm{CO}_{2}$ emission rights, as a net-buyer of emission rights will lose from participating in such a trade. 
